\title{
Differential recall of problems, clues, and solutions from completed and uncompleted tasks
}

\author{
MELVIN H. MARX, EDWARD J. PAVUR, JR., and GEORGE E. SEYMOUR \\ University of Missouri, Columbia, Missouri 65201
}

\begin{abstract}
Forty college students were given the task of solving 28 anagrams with the help of clues. The anagrams were constructed so that problem words, solutions, and clues were common English words. Set was varied between the two groups by telling one group to expect a recall test (open set) and not so informing the other group (closed set). Clues leading to solutions were subsequently recalled more frequently. Subjects in the open group tended to recall more problem words, while those in the closed group tended to recall more clues. The results were interpreted as being due to differential effects of reward.
\end{abstract}

The present experiment is a replication and extension of earlier work (Bottenberg, Marx, \& Pavur, 1976) in which solution of paper and pencil problems led to better recall of problem clues compared to recall of problem clues from unsolved problems. That experimental design was replicated in this study, using different materials (anagrams).

Like the earlier study, the present experiment was intended to relate the Zeigarnik effect to the broader field of learning. Previous experiments (e.g., Zeigarnik, 1927) have indicated that recall is aided by the persistence of motivation as an outcome of interruption (Lewin, 1935). On the other hand, our recent experiment (Bottenberg et al, 1976) has indicated that recall is aided by motivation reduction (reward, or "reinforcement") as an outcome of completion. This apparent contradiction was interpreted with respect to the nature of the tasks used. Experiments demonstrating the classic Zeigarnik effect have typically used performance-type tasks, such as naming a specific number of authors, letter cancellation, and connecting dots. Our results were obtained with a problem-type task, which requires a relatively new organization of behavior in order to attain an unforeseeable solution.

The present experiment was designed to use problemtype tasks. Thus, preferential recall due to completion, rather than to interruption, was predicted. Unlike the earlier study, which used a variety of different tasks requiring irregular time intervals, the present experiment used more homogeneous materials, allowing additional control over the presentation rate, the nature of the interruption, and the instructional set.

This research was supported in part by Research Career Award 1-K6-MH-22-023 from the National Institute of Mental Health and by Grant DAHC19-74-G-0008 from the U. S. Army Research Institute for the Behavioral and Social Sciences to the first author. The opinions stated in this report are those of the authors and are no way to be considered as endorsed by the U. S. Army. The second author is now at the University of New Orleans, New Orleans, Louisiana 70122.
This study manipulated the instructional variable in order to determine some of the boundary conditions within which completion leads to improved recall. One group of subjects was not informed that they would later be tested for recall (closed set), while the other group was informed that they would later be asked to recall problems, clues, and solutions (open set).

\section{METHOD}

\section{Subjects}

Forty students, 23 males and 17 females, from an introductory psychology course received class credit for participating. A group testing procedure was used; the first group was given a closed instructional set, whereas the second was given an open set.

\section{Materials}

The problems used were five-letter anagram words whose letters could be rearranged to form another word. The anagrams were selected from those used by Rees and Israel (1935). The anagram presented to the subject was called the "problem," and the word which the subject was trying to form was called the "solution." For each problem-solution pair another word was selected as a clue to the correct solution. The clues were syntagmatic associates; that is, when combined with the solution, they made up a common or colloquial expression such as "lost cause" or "cheap skates."

\section{Procedure}

The subjects were given an answer sheet numbered from 1 to 28 , and were informed that the experiment involved problem solving. They were told: (1) that they would be given anagram problems to solve, (2) what anagrams were, (3) the nature of the clues, (4) how many problems would be presented, and (5) how much time they would have to solve each one. They were also instructed to solve each problem mentally, and were asked not to write anything on their answer sheet except the solutions. The open-set group was additionally informed that they would later be tested for recall of problems, clues, and solutions.

All subjects were then shown two four-letter problems and clues as examples. It was pointed out that when the clue preceded the problems, as in one example, it meant that the clue was the first word of the phrase; when the clue followed the problem, as in the other example, the solution was the first word of the phrase. The way in which the clues and problems went together was then demonstrated by examples on a blackboard. 
The subjects were reminded that the clues were in parentheses and that the problems were not. Then the experimenter asked if there were any questions, and answered any briefly.

The problem-clue word pairs were typed in lowercase on slides and were presented by a slide projector at a 15 -sec rate per slide. After the last slide had been presented, the answer sheets were collected. The subjects then received another sheet and were given $5 \mathrm{~min}$ to recall, in any order, as many of the problems, clues, and solutions as they could.

\section{RESULTS}

To analyze the number of words recalled, a mixed 2 by 2 by 2 by 2 design was used, the between-subjects variables being set (open vs closed) and sex, and the within-subjects variables being outcome (solved vs unsolved) and word (problem vs clue). Because of the unequal numbers of subjects per cell, a linear regression analysis was performed, providing a general least-squares solution (Winer, 1962).

Tables 1 and 2 present the recall means for clues and problems as a function of outcome and word type and set and word type, respectively.

Reliable main effects were outcome and word. Words from problem-clue slides were recalled more often if the subject had solved that particular problem than if it had not been solved, with the mean number of words recalled being 2.40 for solved and 1.41 for unsolved problems $[F(1,36)=4.89, p<.03]$. Furthermore, reliably more clues than problem words were recalled, the respective means being 2.74 and $1.08[F(1,36)=39.67$, $\mathrm{p}<.001]$. The main effects of sex and set were not reliable $[\mathrm{F}(1,36)<1]$.

The Outcome by Word interaction was highly reliable $[F(1,36)=16.93, p<.001]$. Problem words were recalled equally well regardless of whether the problems were solved or unsolved $[F(1,36)<1]$, but clues from solved problems were recalled more frequently $[F(1,36)=40.36, p<.001]$.

The Word by Set interaction was reliable $[F(1,36)=$ $6.86, \mathrm{p}<.02]$. Subjects in the closed group recalled reliably more clues than subjects in the open group $[F(1,36)=4.19, \mathrm{p}<.05] ;$ there was a tendency for subjects in the open group to recall more problems than subjects in the closed group $[\mathrm{F}(1,36)=3.72, \mathrm{p}<.06]$.

Table 1

Mean Number Recalled as a Function of Outcome and Word Type

\begin{tabular}{lcc}
\hline & \multicolumn{2}{c}{ Outcome } \\
\cline { 2 - 3 } Word & Unsolved & Solved \\
\hline \multirow{2}{*}{ Clue } & 1.750 & 3.725 \\
\multirow{2}{*}{ Problem } & $(1.765)$ & $(2.331)$ \\
& 1.075 & 1.075 \\
& $(1.328)$ & $(1.403)$ \\
\hline
\end{tabular}

Note-Standard deviations are in parentheses. Each cell is based on the data from 40 subjects.
Table 2

Mean Number Recalled as a Function of Set and Word Type

\begin{tabular}{lcc}
\hline & \multicolumn{2}{c}{ Set } \\
\cline { 2 - 3 } Word & Open & Closed \\
\hline \multirow{2}{*}{ Clue } & 2.353 & 3.022 \\
& $(2.308)$ & $(2.246)$ \\
Problem & 1.441 & .804 \\
& $(1.655)$ & $(1.024)$ \\
\hline
\end{tabular}

Note-Standard deviations are in parentheses. The open cells are based on the data from 17 subjects, and the closed cells are based on the data from 23 subjects.

Finally, there was a marginally reliable Outcome by Sex interaction $[F(1,36)=3.74, p<.06]$. There was no sex difference in the recall of solved problems $[\mathrm{F}(1,36)<1]$, but males tended to recall fewer words from unsolved problems $[F(1,36)=3.65, p<.06]$. No other higher order interactions approached reliability.

\section{DISCUSSION}

The finding that clues from solved problems were recalled more frequently than clues from unsolved problems replicates the previous results (Bottenberg et al., 1976). This outcome, like that previously reported, can be accounted for by assuming differential effects of reward. In a problem-type task, a clue which is instrumental in obtaining a solution is more likely to be recalled than a clue which is not instrumental in obtaining a solution. There was no effect of problem solution on the recall of problems. Reward may have exerted a more powerful effect in the recall of the clues as a result of the instrumental role they assumed when leading to problem solution.

Additional support for this interpretation comes from the interaction of word with instructional set. If the completion of the problem is rewarding, and if reward "stamps in" response, changing from a closed to an open instructional set should moderate the reinforcement effect, because an open-set task is not subjectively completed until the recall phase has ended. An analogous finding was reported by Marrow (1938), who used a performance-type task. Marrow instructed his subjects that he would interrupt tasks in which the subjects demonstrated sufficient mastery. Thus, interruption signified subjective success, and completion signified unsuccessful performance. Marrow found that the completed (unsuccessful) tasks were better recalled.

In the present study, the use of problem-type materials was expected to lead to different results. The reinforcing effect of task completion was indeed diminished with the open instructions. Thus, although informing subjects of a later recall test seemed to improve recall of noninstrumental problem words, it also reduced the number of instrumental clue words which were recalled. The former effect may be attributed to differential attention in the open and closed instructional conditions, on the assumption that most college students instructed to recall words tend to concentrate on the problems themselves unless specifically directed to the clues. The latter effect may be partly due to an attentional tradeoff and partly to the unavailability of subjective completion, and thus "reinforcement," in the open condition.

\section{REFERENCES}

Bottenberg, R. A., Marx, M. H., \& Pavur, E. J. Differential recall of problem names and clues as a function 
of problem solution or nonsolution. Bulletin of the Psychonomic Society, 1976, 7, 445-448.

LEwin, K. A dynamic theory of personality. (D. K. Adams \& K. E. Zener, trans.). New York: McGraw-Hill, 1935.

MARrow, A. J. Goal tensions and recall: II. Journal of General Psychology, 1938, 19, 37-64.

REES, H. J., \& IsRaEL, H. E. An investigation of the establishment and operation of mental sets. Psychological Monographs, 1935, 46, Nọ. 210.
WINER, B. J. Statistical principles in experimental design. New York: McGraw-Hill, 1962.

ZeIGARNIK, B. Das Behalten erleidigter und unerleidigter Handlungen. Psychologische Forschungen, 1927, 9, 1-85.

(Received for publication December 9, 1976.) 